\title{
La kinésithérapie en libéral chez les enfants porteurs de maladies neuromusculaires : Vous avez dit kiné ? Pourquoi, quand, comment?
}

\author{
Stéphanie Gilabert
}

Dans le cadre de consultation neuromusculaire, une question des parents revient souvent. "Combien de fois mon enfant doit-il être vu en kiné ? Est-ce que le kiné fait ce qu'il faut?".

Et à l'inverse lorsque je demande à un enfant suivi depuis longtemps : "Pourquoi vas-tu chez le kiné ? À quoi cela sert-il ?", j'obtiens peu de réponses.

$\mathrm{Au}$ fil de discussions entre les enfants et les kinésithérapeutes libéraux, un problème ressort: aucun des deux n'a d'objectif défini. Ni le kinésithérapeute, ni le patient!

Etre porteur d'une maladie neuromusculaire, chronique, stable ou dégénérative, n'empêche pas de déterminer des buts précis, adaptés au handicap. Qui pourrait adhérer à un traitement régulier et parfois contraignant sans avoir d'objectif?

Chaque prise en charge varie en fonction de nombreux critères : le type d'atteinte de l'enfant, l'âge, la croissance, la saison. Tout est à moduler régulièrement en fonction de la situation.

$\mathrm{Au}$ vu des différents articles, de plus en plus fréquents, sur l'histoire naturelle des maladies neuromusculaires, nous pouvons définir des objectifs et agir de manière préventive face à certaines complications indéniables. Dans d'autres cas, c'est l'espoir d'un essai thérapeutique ou d'un traitement innovant qui modifie notre prise en charge. Et dans tous les cas, c'est l'écoute, l'échange avec le patient et sa famille qui permettra de fixer et renouveler de nouveaux objectifs pour avancer ensemble dans l'accompagnement de la maladie.

Il est évident que le manque de connaissances par les kinésithérapeutes libéraux concernant les maladies neuromusculaires limite les objectifs de prise en charge. C'est à nous, kinésithérapeutes hospitaliers de mieux communiquer avec eux et de les aider à progresser dans la connaissance de ces pathologies rares. La question qui me revient de leur part est : "Est ce qu'il va s'améliorer?".

Commencer par réaliser un bilan initial précis, même lorsqu'on travaille en libéral, me semble indispensable. Ensuite, pour affiner nos objectifs et nos choix de traitement, je vous propose de répondre aux questions suivantes: Pourquoi? Combien de fois? Comment? Où?

Un conseil aux kinésithérapeutes et aux familles: posez-vous et notez par écrit les réponses à ces questions. Mettez-les en commun pour être sûr que vous allez dans la même direction.

\section{Pourquoi?}

Et oui pourquoi? "Pourquoi fait-on de la kinésithérapie à mon enfant? Dans quel but? De toute manière, il ne marche plus..." ". Même si les objectifs ne sont peut-être pas ceux dont rêve la famille ou le patient, ils permettent d'avancer.

\section{Différents exemples d'objectifs :}

- Faire en sorte que l'enfant soit le plus confortable possible, lutter contre les douleurs.

Ici, on ne vise que le bien-être de l'enfant. Dans certaines pathologies dégénératives, c'est parfois le seul objectif que l'on peut avoir. Certains parents souhaiteraient qu' " on en fasse plus " pour un enfant en accompagnement de fin de vie par exemple. Le kiné lui va viser le confort. Si ce n'est pas clair pour les parents, ils garderont l'impression que leur enfant n'a pas bénéficié de ce qu'il "fallait".

- Prévenir ou lutter contre les déformations articulaires qui pourraient survenir avant qu'elles ne s'installent comme les rétractions des triceps suraux dans la dystrophie de Duchenne.

- Maintenir la mobilité articulaire actuelle même s'il y a une déformation, ou qu'il ne marche plus, l'enfant ne doit pas avoir de flessums de genoux trop important pour qu'il puisse tenir assis sans douleur, sans traction permanente. Ou qu'il puisse continuer à porter ses chaussures préférées (Figure 1).

- Se préparer le plus efficacement possible sur le plan respiratoire et orthopédique à l'arthrodèse vertébrale : Si le patient est à l'approche de l'arthrodèse vertébrale, oui, il va être important de ne pas lâcher, de trouver la motivation et peut-être 


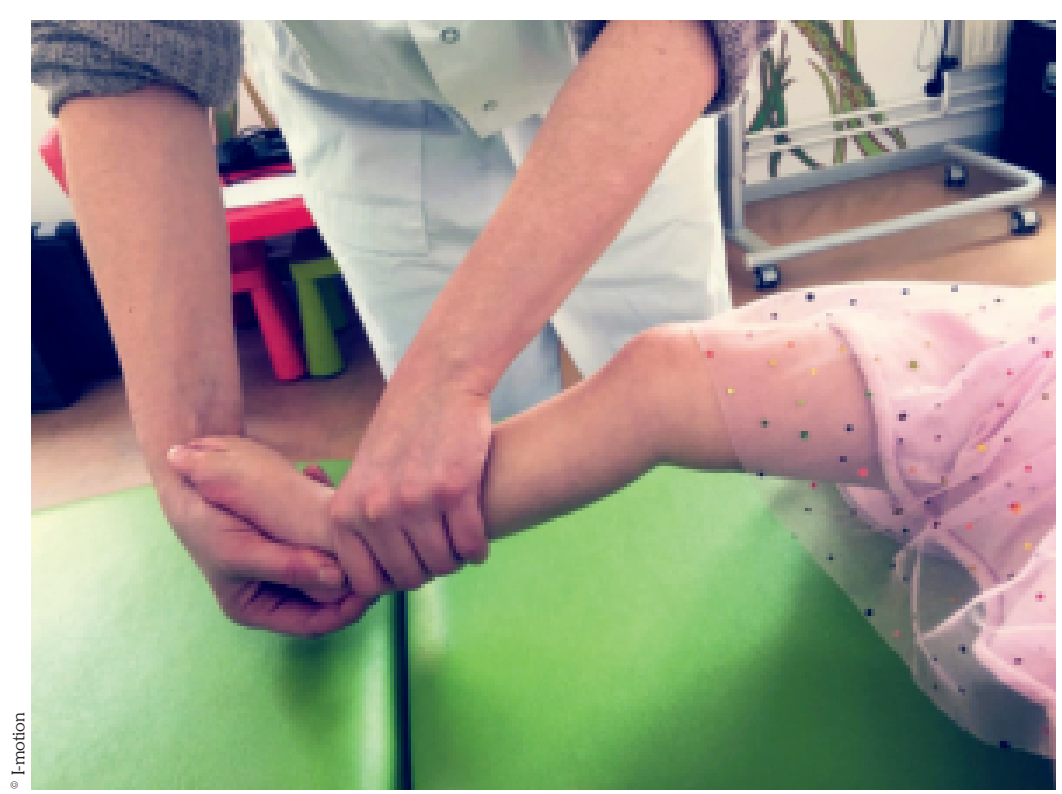

Figure 1

La mobilisation articulaire chez l'enfant.

d'augmenter le nombre de séances de kiné, d'augmenter la durée des séances d'alpha 300 pour se préparer au mieux à cette lourde intervention.

- Prévenir le risque d'infection respiratoire/ éviter d'être hospitalisé. Si nous sommes en hiver, l'intensification des séances de percussionaire et ou d'Alpha 300 sera nécessaire. Si on définit un objectif précis et une durée donnée à l'enfant, cela permettra de passer la période à risque avec une meilleure adhésion aux machines respiratoires et de pouvoir relâcher un peu pendant les vacances d'été par exemple.

Il existe de nombreux autres objectifs.

En résumé, trouvez ensemble le POURQUOI des séances de kinésithérapie du moment.

\section{Combien de fois?}

"Une, deux, trois fois par semaine? Est-ce qu'il en fait assez?".

Avec un "pourquoi " clair, il sera plus simple de savoir combien de fois, il est nécessaire de voir l'enfant. Et le nombre de séances n'est pas figé. Selon les périodes, en fonction du bilan, elles peuvent être augmentées ou diminuées.

Quelques exemples : La réalisation d'une chirurgie va nécessiter d'intensifier la prise en charge. La période d'hiver va demander d'être plus actif sur la prise en charge respiratoire. À l'inverse si l'enfant n'est pas en poussée de croissance et que ses déformations sont stables depuis plusieurs mois, on peut envisager de diminuer le nombre de séances. Faire faire trois séances de kinésithérapies par semaine à un enfant qui n'en a pas besoin, est le meilleur moyen de le démotiver. Il se rend bien compte que "le kiné fait toujours la même chose".

Pour certaines prises en charge où l'on espère une amélioration de la force motrice, il est également nécessaire de donner un délai pour voir le bénéfice ou non de ce qui est mis en place: $\mathrm{X}$ nombre de séances par semaine pour $\mathrm{Y}$ temps. Chez un enfant qui n'a eu aucune prise en charge, on est en droit d'attendre une amélioration, ne serait-ce que sur son endurance musculaire.

On part par exemple sur trois séances, trois fois par semaine, pendant trois mois. Et on fait le point au bout de trois mois. Avec nos téléphones, on peut prendre des photos, filmer la démarche d'un enfant et lui montrer objectivement l'intérêt des exercices/ étirements. Quelle meilleure motivation pour lui de se rendre compte par lui-même qu'il boite moins sur une vidéo?

Si notre traitement ne fonctionne pas, cela nous permettra de l'adapter.

Faites un carnet d'objectif avec votre patient et trouvez ensemble un objectif réalisable à atteindre dans trois mois. Certains objectifs simples peuvent être établis comme par exemple : "Gagner $10^{\circ}$ sur le flessum de genou pour rendre la verticalisation moins douloureuse."

Et pendant ces trois mois, l'enfant s'engage à être assidu dans ses séances de rééducation et à ce qu'on lui demande en dehors. Faites signer un contrat à l'enfant en plus de ce carnet d'objectifs et il y a des chances qu'il adhère mieux à ce traitement. Et refaites le point ensemble tous les trois mois par exemple.

\section{Comment?}

De quoi doivent être composées les séances de kinésithérapie? Les parents posent souvent cette question. "Que doit faire le kiné?".

Les moyens disponibles en kinésithérapie sont nombreux. Le basique indispensable reste les mobilisations des quatre membres, du rachis cervical et du tronc ainsi que le travail respiratoire (avec l'Alpha 300, le percussionaire...). Mais comme le reste, tout est modulable.

En fonction du bilan que le kiné se doit de faire régulièrement, les priorités sont ciblées.

Lorsque l'enfant marche encore, il est indispensable de lui apprendre des auto-étirements.

Former à certains étirements, l'autonomiser sur certains exercices, peut permettre de supprimer une séance dans la semaine et de responsabiliser l'enfant, le rendre actif et acteur. Trop de fois lors de consultations, je demande à l'enfant ce que fait 
son kinésithérapeute libéral et j'entends : "il me masse et m'étire et moi, je reste allongé sur la table". Passif...

Pour la prise en charge orthopédique, les mains du kiné 3 fois 30 minutes par semaine sont insuffisantes face à des rétractions qui s'installent 24h/24. La kinésithérapie doit être couplée aux appareillages. Cela passe, pour les membres inférieurs, par des attelles de nuit anti-équin, des attelles de membres inférieurs, une installation en matelas moulé... Si le kinésithérapeute mobilise l'enfant, mais que la nuit, il est mal installé, cela n'a pas de sens. Tout doit être complémentaire.

Pour ce rôle-là, l'adhésion des parents est indispensable. Eux-aussi doivent devenir des acteurs responsables. Emmener l'enfant trois fois par semaine chez le kiné, c'est bien. Et en même temps, lui faire porter ses attelles huit heures par nuit, c'est mieux ! Ils font partie du " comment " de la bonne prise en charge de l'enfant. Parfois, ils ne se rendent pas compte de cela. Le kinésithérapeute libéral a un rôle indispensable pour être sûr que tout se passe bien en termes d'appareillage à la maison. Il peut lui ou les parents transmettre les informations en cas de difficultés.

Pour la prise en charge respiratoire : l'Alpha 300 est souvent utilisé par les parents. Il reste important que le kiné (libéral ou de la consultation pluridisciplinaire) vérifie que les paramètres et les réglages sont adaptés, que l'enfant le réalise bien, que le thorax se soulève bien, que la gaine soit encore à la bonne taille...

En bref, le kinésithérapeute, les parents et l'enfant doivent agir main dans la main pour une meilleure prise en charge.

Un autre outil de rééducation est l'activité physique en piscine : c'est l'activité idéale pour les amyotrophies spinales par exemple. Que ce soit en balnéothérapie avec le kiné qui en profitera pour mobiliser l'enfant ou en piscine avec les parents. L'enfant est plus libre de ses mouvements, il peut bouger facilement.

Pour la respiration, d'autres pistes ludiques peuvent être utilisées. Cela ne dispensera pas du relaxateur de pression mais ce peut être un bon complément : par exemple, les instruments de musique à vent, le chant, le théâtre, la sarbacane.

Quand cela est possible, le choix d'une activité extrascolaire, adaptée au handicap de l'enfant est essentiel pour lui permettre de développer une meilleure image de lui. N'est-il pas plus sympa de dire de temps en temps aux copains et copines : "Je vais au foot fauteuil" ou "je vais au cours de chant" plutôt que "je vais chez le kiné". Je suis sûre que l'enfant fera d'autant mieux sa rééducation respiratoire si cela peut améliorer sa façon de chanter à la chorale.

\section{...et pour finir : où}

Certains parents préfèrent que le kinésithérapeute vienne à domicile. Cela est plus confortable pour eux. Malheureusement, le kiné ne possède pas les mêmes outils à domicile que ceux dans son cabinet. Les séances sont souvent plus courtes à domicile. Peut-être, faudrait-il envisager d'alterner une séance sur deux ? En revanche, lors des périodes hivernales, il est plus prudent que le kiné vienne à domicile. D'autant plus qu'il y a beaucoup de bronchiolites dans les salles d'attente.

Pour ça aussi, il est important d'expliquer les choix pris avec les bénéfices et les risques.

Pour finir, Il n'y a pas de recette miracle, pas de traitement type. Chaque enfant est unique et la prise en charge est à moduler en fonction de nombreux paramètres. Certes, ce sont des maladies lourdes, souvent dégénératives. Et, c'est exactement pour cela que les parents et les enfants doivent sentir que l'ont fait au mieux pour eux en dépit de la maladie. Posez-vous ensemble : enfant, parents, kinés. Discutez. Définissez des objectifs et notez tout cela dans un carnet commun pour partir sur de bonnes nouvelles bases!

Private practice of physiotherapy in children with neuromuscular diseases: did you say physiotherapy? Why, when, how?

\section{LIENS D'INTÉRÊT}

L'auteur déclare n'avoir aucun lien d'intérêt concernant les données publiées dans cet article. 